\title{
83. COMPORTAMIENTO FITOSOCIOLÓGICO DE MEDICAGO CITRINA (FONT QUER) GREUTER (LEGUMINOSAE), ENDEMISMO MEDITERRÁNEO- IBEROLEVANTINO
}

\author{
Ana JUAN y Manuel B. CRESPO
}

\begin{abstract}
Phytosociological behaviour of Medicago citrina (Font Quer) Greuter (Leguminosae), an IberolevantineMediterranean endemic
\end{abstract}

Palabras clave. Medicago, Beta, vegetación nitrófila, Medicagini-Lavaterion arboreae, endemismos.

Key Words. Medicago, Beta, nitrophilous plant communities, Medicagini-Lavaterion arboreae, endemics.

Medicago citrina (Font Quer) Greuter es una leguminosa arbustiva endémica del territorio mediterráneo-iberolevantino (sensu Rivas Martínez, 1987). Su distribución queda restringida a medios insulares de la Comunidad Valenciana (Parque Natural de las Islas Columbretes) y de las Islas Baleares (Ibiza y Parque Nacional de Cabrera) (Bolòs y Vigo, 1990a; Bibiloni et al., 1993; Juan et al., 1999). Este endemismo se desarrolla en ambientes litorales, donde el hálito marino tiene una fuerte influencia, y donde, además, se acumulan en el suelo depósitos de desechos orgánicos (guano) resultantes de la actividad de las aves marinas que viven en tales ambientes. Esta planta está considerada "en peligro de extinción" (Catálogo Nacional de Especies Amenazadas, Real Decreto 439/1990); aunque recientemente Laguna y Crespo (1996) han propuesto catalogarla en la categoría "Vulnerable" (VU), según las directrices de la U.I.C.N. (noviembre de 1994).

Las formaciones vegetales dominadas por M. citrina fueron descritas por Bolòs y Vigo (1984) bajo el nombre Medicagini citrinaeLavateretum arboreae, a partir de un inventario levantado en el Archipiélago de las Columbretes. Posteriormente, Bolòs (1989) aportó nuevos inventarios, también procedentes de las Columbretes. Florísticamente, esta asociación se caracteriza fundamentalmente por la presencia de los elementos arbustivos M. citrina y Lavatera arborea L., que encuentran su óptimo en ambientes litorales donde la nitrificación por guano y la salinidad que aporta el hálito marino son patentes. Además, la comunidad suele enriquecerse con otros elementos perennes nitrófilos y nitrohalófilos tales como Suaeda vera Forssk., Beta maritima L. (s.1.) o Daucus gingidium L. subsp. commutatus (Paol.) O. Bolòs \& Vigo, en mosaico con diversas formaciones nitrófilas anuales. Con posterioridad, Rita y Bibiloni (1993) estudiaron formaciones arbustivas análogas de las islas Baleares, donde tienen una buena representación en los islotes que rodean Cabrera e Ibiza. Estos autores encontraron cierta variabilidad florística en sus inventarios debido a la presencia de Beta vulgaris L. var. marcosii O. Bolòs \& Vigo, taxon propio de los islotes baleáricos (cf. Bolòs y Vigo, 1990a), que aquí se considera en el rango de subespecie. Por ello, propusieron una nueva subasociación-Medicagini-Lavateretum arboreae subass. betetosum marcosii-, vicariante baleárica de la típica columbretense.

\section{Ecología y fitosociología}

En el marco de las investigaciones que se están realizando sobre el endemismo $M$. citrina (cf. Juan et al., 1999), se ha llevado a cabo un 
estudio fitosociológico de sus poblaciones en la totalidad de su área de distribución. La comparación de los inventarios levantados en las poblaciones columbretenses y baleáricas pone de manifiesto la existencia de notables diferencias florísticas, ecológicas, catenales y biogeográficas, que permiten reconocer dos sintáxones diferentes, como hicieron Rita y Bibiloni (1993); aunque ambos con el rango de asociación.

\section{Medicagini citrinae-Lavateretum arboreae $\mathrm{O}$.}

Bolòs, Folch et Vigo in O. Bolòs y Vigo 1984 [Els sistemes Naturals de les Illes Medes: 184-185]

Holotypus: O. Bolòs y Vigo, loc. cit.: 185, inventario único

Bosquetes densos de Medicago citrina, al que acompañan, en mayor o menor medida, Lavatera arborea y Suaeda vera. Actúan como elementos característicos y diferenciales Lobularia maritima (L.) Desv. subsp. columbretensis R. Fern., Reseda hookeri Guss. o Sonchus tenerrimus L. var. dianae (Lacaita ex Willk.) O. Bolòs \& Vigo, además de otras plantas de distribución más amplia como Patellifolia patellaris (Moq.) A.J. Scott et al. (Beta patellaris Moq.), Lavatera mauritanica Durieu [incl. subsp. davaei (Cout.) Cout.] o Beta maritima subsp. maritima, que permiten reconocer igualmente la asociación (tab. 1). En sus claros se desarrollan herbazales anuales nitrófilos de Chenopodion muralis Br.-Bl. 1931, muy pobremente caracterizados (cf. Bolòs y Vigo, 1984), o comunidades hemicriptofíticas de Bromo-Oryzopsion miliaceae O. Bolòs 1970 (Ass. Euphorbio terracinae-Lobularietum columbretensis Carretero y Boira 1987, corr. Carretero y Aguilella 1995).

Se instalan sobre litosuelos pliocuaternarios de origen volcánico -principalmente basaltos- (Alonso, 1987), bajo un bioclima termomediterráneo semiárido, aunque localmente el rocío matinal y, en ocasiones, las nieblas contribuyen a aumentar la humedad ambiental. Actualmente, las mejores representaciones de esta asociación se encuentran en las islas Ferrera y Foradada, en el Archipiélago de las Columbretes (sector Valenciano-Tarraconense, provincia CatalanoValenciano-Provenzal), de donde es endémica.

Catenalmente, la Medicagini-Lavateretum contacta con la Dauco commutati-Sonchetum dianae $\mathrm{O}$. Bolòs, Folch et Vigo in O. Bolòs 1989 (Crithmo-Staticion Molinier 1934) en la base de los acantilados, donde la influencia del oleaje es manifiesta; mientras que hacia las zonas altas de los acantilados, donde la influencia del oleaje es menor, puede entrar en contacto (e.g. en la Ferrera) con fragmentos de vegetación serial de la Chamaeropo humilisRhamnetum lycioidis O. Bolòs 1957 (Al. Asparago albi-Rhamnion oleoidis Rivas Goday 1964, em. Rivas Mart. 1975).

Beto marcosii-Medicaginetum citrinae (Rita y Bibiloni 1993) A. Juan y M. B. Crespo, comb. et stat. nov.

Basion.: Medicagini citrinae-Lavateretum arboreae betetosum marcosii Rita y Bibiloni 1993 [Història Natural de l'Arxipèlag de Cabrera: 241-243]

Holotypus: Rita y Bibiloni, op. cit.: 239, tab. 11 , invent. 2

Bosquetes densos de Medicago citrina, en los que Lavatera arborea y Suaeda vera participan de modo variable, dando un aspecto muy similar al de la asociación anterior. Florísticamente, queda bien independizada de su vicariante columbretense por la presencia de elementos baleáricos o diánico-baleáricos como Beta maritima subsp. marcosii (O. Bolòs \& Vigo) A. Juan \& M. B. Crespo, Senecio leucanthemifolius Poir., Diplotaxis ibicensis (Pau) Gómez-Campo o Allium commutatum Guss. Además, esta asociación puede enriquecerse localmente con otros elementos 
Tabla 1

Medicagini citrinae-Lavateretum arboreae O. Bolòs, Folch et Vigo

in O. Bolòs y Vigo 1984

(Medicagini-Lavaterion, Salsolo-Peganetalia, Pegano-Salsoletea)

$\mathrm{N}^{\circ}$ de orden

Área $\left(\mathrm{m}^{2}\right)$

Exposición

Altitud (m)

Cobertura (\%)

$\begin{array}{ccc}1 & 2 & 3 \\ 100 & 50 & 150 \\ 45 \mathrm{NW} & 30 \mathrm{SW} & 35 \mathrm{~N} \\ 55 & 50 & 45 \\ 80 & 95 & 90\end{array}$

4
100
$45 \mathrm{NW}$
35
100

\section{5}

150

$35 \mathrm{NW}$

40

100

\section{6}

25

$35 \mathrm{NW}$

$-$

85

Caract. asociación y unid. sup.

Medicago citrina

Lavatera arborea

Reseda hookeri

Sonchus tenerrimus var. dianae

Lavatera mauritanica

Suaeda vera

Beta maritima subsp. maritima

Patellifolia patellaris

\section{Compañeras}

Silene latifolia

Daucus gingidium subsp. commutatus

Silene sclerocarpa

Chenopodium murale

Lobularia maritima subsp. columbretensis

Asparagus horridus

Merculiaris ambigua

Parietaria judaica

Succowia balearica

Brachypodium distachyon

Hyoscyamus albus

Smilax aspera

Lamarckia aurea

$\begin{array}{cccccc}2 & 4 & 4 & 5 & 4 & 1.2 \\ 4 & 3 & 2 & 2 & 3 & 3.1 \\ . & 1 & + & + & . & . \\ + & . & . & . & 1 & + \\ 2 & . & 1 & . & . & . \\ 1 & . & . & . & 2 & . \\ . & . & + & . & . & + \\ . & + & . & . & 1 & .\end{array}$

Procedencia de los inventaios: 1.- Columbrete Grande, zona $\mathrm{N}$ del faro. 2., 3. y 4.- Foradada. 5.- Ferrera. 6.- Foradada, holotypus (Bolòs y Vigo, 1984). Todos del Archipiélago de las Columbretes (Castellón).

como Limonium pseudebusitanum Erben o $L$. caprariense (Font Quer \& Marcos) Pignatti, que actúan como buenas diferenciales territoriales del sintaxon balear (tab. 2). En sus claros se desarrollan, igualmente, herbazales anuales nitrófilos de Chenopodion muralis.

Se instalan sobre litosuelos calcáreos mesozoicos (Alcover, 1993), bajo un bioclima termomediterráneo seco-semiárido, muy suavizado por la frecuencia del rocío y las nieblas. Esta asociación se encuentra bien constituida en los islotes cercanos a Ibiza (Ses Malvins, S'Espartar y Na Bosc) y Cabrera (Estell de Coll, Estell de Fora y Ses Bledes), en 
la provincia Balear, de donde es endémica.

Catenalmente, la Beto-Medicaginetum suele contactar con comunidades de CrithmoStaticion, en los acantilados salpicados por la maresía, y con fragmentos empobrecidos de maquias de Oleo-Ceratonion Br.-B1. 1936 ex Guinochet y Drouineau 1944 (e.g. Malví Gros), en las zonas más protegidas.

No obstante, en la tabla 2 se observa que existen interesantes diferencias florísticas entre los inventarios procedentes de Ibiza y los de Cabrera. Ello permite reconocer dos subasociaciones, que responden a cambios bioclimáticos y biogeográficos, que tiene una traducción incluso en aspectos catenales como se indica a continuación:

betetosum marcosii (tab. 2, invent. 1-4): la típica, bastante pobre en especies; aunque la presencia de Limonium caprariense, endemismo gimnésico (Cabrera y sur de Mallorca) permite reconocerla fácilmente. Se trata de un sintaxon endémico del sector Mallorquín (subprovincia Gimnésica). Bioclimáticamente se desarrolla bajo bioclima termomediterráneo seco y catenalmente contacta, a menudo, con la Limonietum caprariensis O. Bolòs y Molinier 1958.

salsoletosum oppositifoliae A. Juan y M.B. Crespo, subass. nov. (tab. 2, invent. 5-7; holotypus: invent. 5): Sintaxon bien caracterizado florísticamente por la presencia de Salsola oppositifolia Desf. y Cynomorium coccineum L., plantas ampliamente representadas en el litoral del sudeste ibérico, que ponen de manifiesto una vez más las estrechas relaciones existentes entre Ibiza y dichos territorios. Resulta endémico del sector Ibicenco (subprovincia Pitiúsica), evidenciando un bioclima termomediterráneo semiárido (similar al de los citados territorios Murciano-Almerienses). Catenalmente, esta subasociación contacta con la Limonietum ebusitani Rivas Mart. et al. 1992 (en la que está ausente Limonium caprariense), lo que refuerza más, si cabe, su independencia frente a la subasociación típica.

\section{Esquema sintaxonómico}

PEGANO HARMALAE-SALSOLETEA

VERMICULATAE Br.-Bl. y O. Bolòs 1958

+ Salsolo vermiculatae-Peganetalia harmalae Br.-Bl. y O. Bolòs 1958

* Medicagini citrinae-Lavaterion arboreae $\mathrm{O}$. Bolòs, Folch et Vigo in $\mathrm{O}$. Bolòs y Vigo 1984

Medicagini citrinae-Lavateretum arboreae $\mathrm{O}$. Bolòs, Folch et Vigo in O. Bolòs y Vigo 1984

Beto marcosii-Medicaginetum citrinae (Rita \& Bibiloni 1993) A. Juan y M.B. Crespo comb. et stat. nov.

\section{betetosum marcosii}

salsoletosum oppositifoliae A. Juan y M.B. Crespo, subass. nov.

Los matorrales halo-nitrófilos dominados por $M$. citrina (tab. 3) se incluyen en la alianza Medicagini citrinae-Lavaterion arboreae descrita por Bolòs y Vigo (1984). Esta alianza agrupa comunidades arbustivas ornitocoprófilas y halófilas, que se desarrollan en acantilados costeros donde se instalan colonias de aves marinas. Presenta su óptimo en los territorios iberolevantinos, bajo bioclimas termomediterráneo seco-semiárido, aunque alcanza el termotipo mesomediterráneo inferior. La combinación florística, ecología y tipo biológico de sus plantas directrices (Medicago citrina y Lavatera arborea) permiten incluirla en la clase fitosociológica Pegano-Salsoletea Br.-Bl. y O. Bolòs 1958, mejor que en el orden Chenopodietalia muralis Br.-B1. in Br.-Bl. et col. 1936 (RuderaliSecalietea Br.-B1. in Br.-B1. et col. 1936) donde había sido incluida inicialmente (Bolòs y Vigo, 1984). Sin embargo, según el esquema 
Tabla 2

Beto marcosii-Medicaginetum citrinae (Rita y Bibiloni 1993)

A. Juan y M.B. Crespo, comb. et stat. nov.

betetosum marcosii (típica)

salsoletosum oppositifoliae A. Juan y M.B. Crespo, subass. nov.

(Medicagini-Lavaterion, Salsolo-Peganetalia, Pegano-Salsoletea)

Orden de inventarios

Área $\left(\mathrm{m}^{2}\right)$

Altitud (m)

Exposición

Cobertura (\%)

$\begin{array}{cc}1 & 2 \\ 30 & 20 \\ 10 & 35 \\ 20 \mathrm{SE} & 20 \mathrm{SE} \\ 70 & 70\end{array}$

3
20
8
-
90

$\begin{array}{cccc}4 & 5 & 6 & 7 \\ 50 & 100 & 50 & 100 \\ 8 & 18 & 9 & 45 \\ - & 10 \mathrm{~N} & - & 5 \mathrm{SW} \\ 75 & 90 & 80 & 90\end{array}$

Caract. asociación y unid. sup.

Medicago citrina

Beta maritima subsp. marcosii

Lavatera arborea

Suaeda vera

Diplotaxis ibicensis

Allium commutatum

Diferenciales subass. salsoletosum

Salsola oppositifolia

Cynomorium coccineum

$\begin{array}{lll}3 & 3 & 5 \\ 2 & 2 & 3 \\ 2 & 1 & . \\ + & . & . \\ . & . & . \\ . & . & .\end{array}$

4
2
.
.
.

$\begin{array}{ccc}4 & 4 & 4 \\ 1 & . & 2 \\ 1 & 1 & 1 \\ 2 & 1 & 2 \\ 1 & 1 & 2 \\ + & . & .\end{array}$

\begin{tabular}{|ccc|}
\hline 2 & 2 & 4 \\
+ & + &. \\
\hline
\end{tabular}

\section{Compañeras}

Asparagus horridus

Limonium pseudebusitanum (dif.)

Chenopodium murale

Silene secundiflora

Limonium caprariense (dif.)

Daucus gingidum subsp. commutatus

Sonchus oleraceus

Senecio leucanthemifolius (dif.)

Sarcocornia fruticosa

Asteriscus maritimus

Mesembryanthemum nodiflorum

Desmazeria marina

Parapholis incurva

Delphinium verdunense

Inula crithmoides

Phagnalon saxatile

Plantago lagopus
Procedencia de los inventarios: 1 y 2.- Estell de Coll (Cabrera). 3.- Ses Bledes (Cabrera). 4.- Illa de ses Bledes (zona central) (Holotypus de la subass. típica, Rita y Bibiloni, 1993). 5.- Es Malví Gros (Ibiza). 6.Es Malví Plà (Ibiza). 7.- Na Bosc (Ibiza). 
actualmente aceptado de la clase PeganoSalsoletea, la alianza Medicagini-Lavaterion debería ubicarse en el orden SalsoloPeganetalia -que agrupa los matorrales halonitrófilos de claro matiz estepario y óptimo en biotipos semiárido y árido-; aunque esta solución resulta un tanto conflictiva. No obstante, parece oportuno tomar esta solución provisionalmente, en espera de disponer de más información derivada de los estudios sintéticos que actualmente estamos realizando sobre este tipo de matorrales ornitocoprófilos y halófilos, en el ámbito de Europa occidental.

\section{Apéndice florístico}

Para la nomenclatura y autorías de los táxones citados se ha seguido, básicamente, a Castroviejo et al. (1986-1999), Bolòs et al. (1990b) y Mateo y Crespo (1998), excepto para el caso siguiente:

Beta maritima subsp. marcosii (O. Bolòs \& Vigo) A. Juan \& M.B. Crespo, comb. et stat. nov.

Basion.: B. vulgaris var. marcosii $\mathrm{O}$. Bolòs \& Vigo, Butll. Inst. Catalana Hist. Nat. 38: 88 (1974)

Las poblaciones de Beta maritima que acompañan a Medicago citrina en los acantilados marinos de las Baleares donde se deposita guano en cantidades apreciables, presentan unas peculiaridades morfológicas que permiten reconocerlas con facilidad. Bolòs y Vigo (1974) propusieron para las plantas de Cabrera (Ses Bledes) la var. marcosii, que diferenciaban por ser "robusta, ramosa, foliosa, seminibus depresso-mamillatis"; posteriormente, Bolòs y Vigo (1990a) reconocían ciertas afinidades entre ésta y la var. perennis L., taxon que Letschert (1993) sinonimizaba, en su monografía del género, con la subsp. maritima (taxon que consideraba muy variable y constituido por numerosas unidades taxonómicas de escaso valor).

Sin embargo, después de estudiar abundante material ibérico de $B$. maritima y compararlo con lo que corresponde a la subsp. marcosii, existen a nuestro juicio caracteres que se mantienen invariables en las poblaciones baleares. Quizás lo más destacable es el hábito de la planta: el tallo principal es muy corto (cesa rápidamente su crecimiento y permanece como una roseta de hojas) y nunca desarrolla inflorescencias, mientras que los laterales, muy numerosos y robustos (con más de $1 \mathrm{~m}$ de longitud), son procumbentes y finalmente se hacen ascendentes portando todos ellos las inflorescencias. Este carácter es apreciable sólo en ejemplares vivos. Por otra parte, los frutos de la subsp. marcosii son apreciablemente mayores (3.5-4.5 mm) que en las formas típicas de la subsp. maritima $(2.5-3.5 \mathrm{~mm})$, lo que puede facilitar el reconocimiento de ejemplares de herbario.

La subsp. marcosii presenta afinidades con algunos táxones del Mediterráneo occidental. Respecto al hábito, existen algunas formas africanas (var. debeauxii Clary) que presentan tallos postrado-ascendentes, aunque sus frutos y semillas son diferentes a los de la subsp. marcosii. Algo similar ocurre con Beta adanensis Pamukçuoglu (endemismo del Mediterráneo oriental), que presenta tallos postrados pero los segmentos periánticos son cortos y poco acrescentes, sin llegar a recubrir el fruto maduro.

Por todo ello, parece apropiado reconocer el taxon balear con el rango subespecífico, el cual se habría diferenciado seguramente por las especiales condiciones ecológicas en que habita (tal vez de modo paralelo a como lo hizo Medicago citrina a partir del agregado de Medicago arborea L.). Según los datos de que disponemos se trata de un endemismo restringido a los islotes cercanos a Cabrera e Ibiza, aunque deberá buscarse en otros ambientes de similares características en las Baleares. 


\begin{tabular}{llllllll}
\hline Orden de inventarios & 1 & 2 & 3 & 4 & 5 & 6 & 7 \\
$\mathrm{~N}^{\circ}$ de inventarios & 5 & 3 & 9 & 3 & 3 & 3 & 1 \\
\hline
\end{tabular}

\section{Características de las asociaciones}

Lobularia maritima subsp. columbretensis

Sonchus tenerrimus var. dianae

Reseda hookeri

Beta maritima subsp. maritima

Lavatera mauritanica

Patellifolia patellaris

Beta maritima subsp. marcosii

Senecio leucanthemifolius

Limonium pseudebusitanum

Diplotaxis ibicensis

Limonium caprariense

Allium commutatum

Salsola oppositifolia

Cynomorium coccineum

\begin{tabular}{|c|c|c|c|c|c|c|}
\hline I & 1 & IV & . & . & . & . \\
\hline II & 2 & III & . & . & - & . \\
\hline III & 2 & I & . & . & . & . \\
\hline I & 2 & II & . & . & . & . \\
\hline $\begin{array}{c}\text { II } \\
\text { I }\end{array}$ & . & . & . & . & . & . \\
\hline . & $\cdot$ & . & 3 & 3 & 2 & 1 \\
\hline · & . & - & · & 1 & 2 & 1 \\
\hline . &. & - & 2 & 3 & 1 & . \\
\hline . & $\cdot$ & - & $\cdot$ & $\dot{\circ}$ & 3 & 1 \\
\hline . & $\cdot$ & . & $\cdot$ & 2 & : & . \\
\hline . & . & - & $\cdot$ & - & 1 & . \\
\hline . & . & . & . & - & 3 & 1 \\
\hline . & . & . & . & . & 2 & . \\
\hline
\end{tabular}

\section{Características de unidades superiores}

Medicago citrina

Lavatera arborea

Suaeda vera

$\mathrm{V}$
$\mathrm{V}$
$\mathrm{II}$

$\begin{array}{ll}\text { IV } & 3 \\ \mathrm{~V} & 1 \\ \mathrm{II} & \cdot\end{array}$

$\begin{array}{lll}3 & 3 & 1 \\ 2 & 3 & 1 \\ 1 & 3 & 1\end{array}$

\section{Compañeras}

Asparagus horridus

Daucus gingidium subsp. commutatus

Chenopodium murale

Silene sclerocarpa

Silene secundiflora

Mesembryanthemum nodiflorum

Crithmum maritimum

Sonchus oleraceus

Silene latifolia

Parapholis incurva

Parietaria judaica

Smilax aspera

Succowia balearica

Mercurialis ambigua

Hyoscyamus albus

Brachypodium distachyon

Lamarckia aurea

Pistacia lentiscus

Sarcocornia fruticosa

Desmazeria marina

Asteriscus maritimus

Inula crithmoides

Phagnalon saxatile

Plantago lagopus

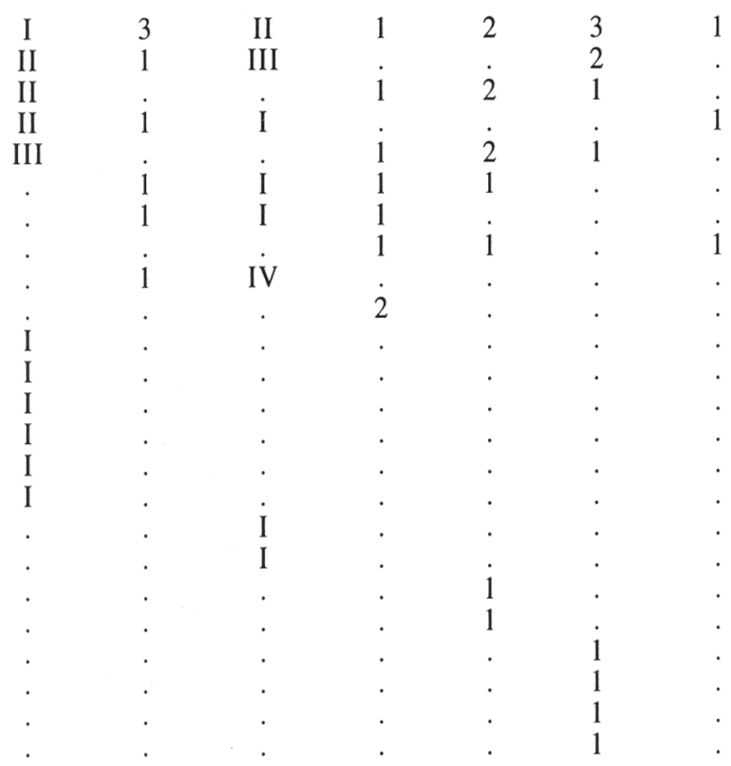

Procedencia de los inventarios: 1.- Medicagini-Lavateretum arboreae (Juan y Crespo, tabla 1, excl. invent. 6). 2.- Medicagini-Lavateretum arboreae Carretero y Boira (1987). 3.- Medicagini-Lavateretum arboreae (Bolòs, 1989). 4.- Medicagini-Lavateretum arboreae betetosum marcosii (Rita y Bibiloni, 1993, tabla 11, invent. 1-3). 5.- Beto-Medicaginetum citrinae betetosum marcosii (Juan y Crespo, tabla 2, invent. 1-3). 6.- Beto-Medicaginetum citrinae salsoletosum oppositifoliae (Juan y Crespo, tabla 2, invent. 5-7). 7.Medicagini-Lavateretum arboreae betetosum marcosii (Rita y Bibiloni, 1993, tabla 11, invent. 4).

Tabla 3. Tabla sintética de las asociaciones dominadas por Medicago citrina (Medicagini-Lavaterion arboreae, Salsolo-Peganetalia, Pegano-Salsoletea) 
Clave de los táxones ibéricos del género Beta L.

1. Plantas anuales. Tallos con flores desde la base. Inflorescencia con brácteas hasta el ápice, brácteas mayores que los glomérulos de flores B. macrocarpa Guss

1'.Plantas sin estos caracteres reunidos 2

2. Planta de hasta $200 \mathrm{~cm}$, con tallos erectos. Hojas 1-3 dm, con el pecíolo muy ancho y de color blanco-marfileño. Brácteas inferiores de la inflorescencia de hasta $2 \mathrm{~cm}$ de longitud, lanceoladas o linear lanceoladas. B. vulgaris $\mathrm{L}$.

2'.Plantas de hasta $110 \mathrm{~cm}$, con tallos postrados o erectos, teñidos con líneas purpúreas. Hojas de hasta $12 \mathrm{~cm}$, con pecíolo más estrecho y nunca blanco-marfileño. Brácteas poco perceptibles, de menos de $2 \mathrm{~cm}$, linear-lanceoladas

B. maritima L.

a. Tallo principal de longitud superior o similar a las ramificaciones laterales, todos erguidos y terminados en inflorescencias. Fruto de 2.5-3.5 $\mathrm{mm}$, semillas globosas, de hasta $1.5 \times 1.2 \mathrm{~mm}$. B. maritima subsp. maritima

a'.Tallo principal muy corto y terminado en roseta foliar, los laterales mucho más largos (frecuentemente de $1 \mathrm{~m}$ de longitud o mayores), floríferos y decumbentes (ascendentes en la floración). Fruto de 3.5-4.5 mm, semillas deprimidas, de $2 \times 1.5 \mathrm{~mm}$

B. maritima subsp. marcosii (O. Bolòs \& Vigo) A. Juan \& M.B. Crespo

AGRADECIMIENTOS. Agradecemos a los guardas del Parque Nacional de Cabrera, del Parque Natural de las Islas Columbretes y de la Conselleria de Medi Ambient del Consell Insular d'Eivissa i Formentera, por su inestimable ayuda en los trabajos de campo. Igualmente a los directores de dichas instituciones, especialmente a Juan Jiménez (Islas Columbretes) por su apoyo durante la realización de nuestras investigaciones. Este trabajo ha sido parcialmente financiado por la Generalitat Valenciana y el Excmo. Ayuntamiento de Castellón.

\section{BIBLIOGRAFÍA}

ALCOVER, J. A., E. BALlesteros y J. J. FORNÓS (eds.) -1989- Història natural de l'Arxipèlag de Cabrera. Consell Superior d'Investigacions Científiques. Ed. Moll. Mallorca.
ALONSO, L. A. -1987-Variaciones petroquímicas en el vulcanismo de las Islas Columbretes. En: Alonso, L. A., J. L. Carretero y M. García Carrascosa (coords.): Islas Columbretes. Contribución al estudio de su medio natural. Colección Monografías, $\mathrm{n}^{\circ}$ 5. Consellería de Obras Públicas, Urbanismo y Transportes. Agència del Medi Ambient. Generalitat Valenciana. Valencia.

BIBILONI, G., G. ALOMAR y J. RITA -1993Flora vascular dels illots i addicions a la flora de Cabrera Gran. En: Alcover, J. A., E. Ballesteros y J. J. Fornós (eds.): Història natural de l'Arxipèlag de Cabrera. Consell Superior d'Investigacions Científiques. Ed. Moll. Mallorca.

BOLÒS, O. -1989- La vegetació d'algunes petites illes properes a la Península Ibèrica. Fol. Bot. Misc. 6: 115-133.

BOLÒS, O. y J. VIGO -1974- Notes sobre taxonomia i nomenclatura de plantes, I. Butll. Inst. Catalana Hist. Nat. 38 (Secc. Bot. 1): 61-89.

BOLÒS, O. y J. VIGO -1984- Flora vascular i vegetació de les Illes Medes. En: Ros, J., I. Olivella, y J. M. Gili (eds.). Els sistemes naturals de les Illes Medes. Institut d'Estudis Catalans. Barcelona.

BOLÒS, O. y J. VIGO -1990a-Flora dels Països Catalans, II. Ed. Barcino. Barcelona.

BOLÒS, O., J. VIGO, R. M. MASALLES y J. M. NINOT -1990b- Flora manual dels Països Catalans Ed. Pòrtic. Barcelona.

CARRETERO, J. L. y H. BOIRA -1987- La vegetación de las Islas Columbretes. En: Alonso, L. A., J. L. Carretero y M. García Carrascosa (coords.): Islas Columbretes. Contribución al estudio de su medio natural. Colección Monografías, $n^{\circ}$ 5. Consellería de Obras Públicas, Urbanismo y Transportes. Agència del Medi Ambient. Generalitat Valenciana. Valencia.

CASTROVIEJO, S. et al. -1986/99-Flora iberica, 8 vols. Real Jardín Botánico, CSIC. Madrid.

JUAN, A., M. B. CRESPO y S. RÍOS -1999Medicago citrina (Font Quer) Greuter (Leguminosae): variabilidad morfológica, ecología y estado actual de sus hábitats. Actas XXXIX Reunión Científ. Soc. Españ. Estud. Pastos (Almería): 87-91.

LAGUNA, E. y M. B. CRESPO-1996- Asignación 
de las nuevas categorías UICN a la flora endémica de la Comunidad Valenciana. Bol. Real Soc. Españ. Hist. Nat. Tomo Extraord. 125 Anivers.: 385-387.

LETSCHERT, P. P. W. -1993-Beta section Beta: biogeographical patterns of variation and taxonomy. Wageningen Agric. Univ. Papers 93(1): 1-155.

MATEO, G. y M. B. CRESPO-1998-Manual para la determinación de la flora valenciana. Monogr. Fl. Montiberica ${ }^{\circ} 3$. Valencia.

RITA, J y G. BIBILONI -1993- La vegetación (Memòria del mapa de les comunitats vegetals). En: Alcover, J. A., E. Ballesteros y J. J. Fornós (eds.): Història natural de l'Arxipèlag de Cabrera. Consell Superior d'Investigacions Científiques. Ed. Moll. Mallorca.
RIVAS MARTÍNEZ, S. -1987-Memoria del mapa de series de vegetación de España 1:400.000. ICONA - Mº Agricultura, Pesca y Alimentación. Madrid.

Aceptado para su publicación en Octubre de 1999

Dirección de los autores. Dpto. Ciencias Ambientales y Recursos Naturales (Botánica). Universidad de Alicante. Apdo. 99. E-03080 Alicante. e-mail: crespo@carn.ua.es

\title{
84. SOBRE PRIMULA HIRSUTA ALL. Y LA NOMENCLATURA DE DOS ASOCIACIONES PIRENAICAS DEL ANDROSACION VANDELLII
}

\author{
José Luis BENITO ALONSO
}

About Primula hirsuta All. and the nomenclature of two pyrenean associations from Androsacion vandellii

Palabras clave. Fitosociología, nomenclatura, Primula hirsuta, Primula latifolia, Androsacion vandellii, España, Francia, Pirineo.

Key words. Phytosociology, nomenclature, Primula hirsuta, Primula latifolia, Androsacion vandellii, Spain, France, Pyrenees.

Primula hirsuta All. y Primula latifolia Lapeyr. son dos primuláceas que pertenecen a la misma sección (Aleurita Duby), por lo que muchos autores las han confundido. En pliego de herbario, $P$. hirsuta se distingue por tener pelos glandulíferos con la célula terminal de color rojizo, mientras que $P$. latifolia los tiene de color pálido, amarillento. En fresco, el color

Trabajo realizado con la financiación del Departamento de Educación y Cultura del Gobierno de Aragón, a través de una beca predoctoral de investigación. 